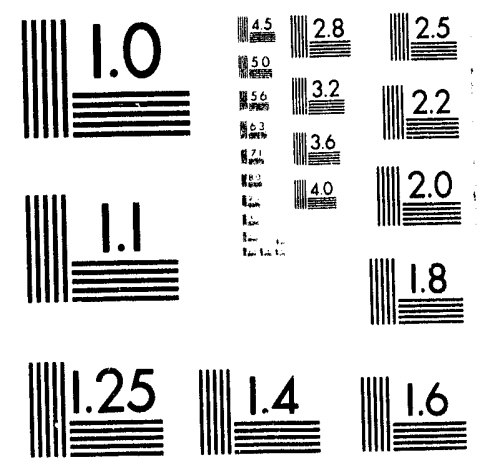



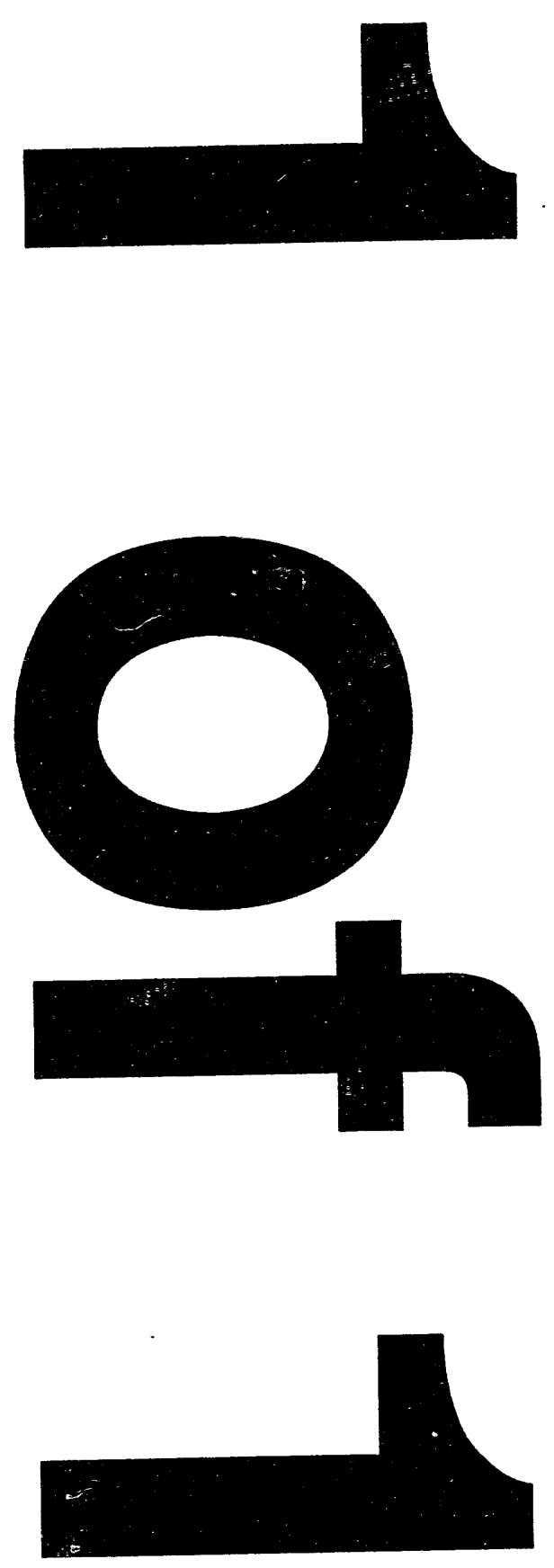


\section{Ballooning-Mirror Instability and Internally Driven Pc 4-5 Wave Events}

C. Z. Cheng, Q. Qian, K. Takahashi ${ }^{1)}$, and A. T. Y. Lui ${ }^{2)}$

Princeton Plasma Physics Laboratory

Princeton University, P. O. Box 451, Princeton, NJ 08543

1) Permanerit address: Solar-Terrestrial Environment Laboratory, Nagoya University, Toyokawa, 442, Japan

2) Permanent address: Applied Physics Laboratory, The Johns Hopkins University, Laurel, MD 20723

\section{MASTER}

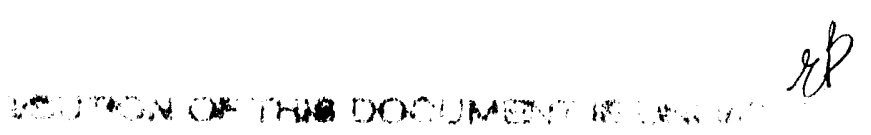


A kinetic-MHD field-aligned eigenmode stability analysis of low frequency ballooningmirror instabilities has been performed for anisotropic pressure plasmas in the magnetosphere. The ballooning mode is mainly a transverse wave driven unstable by pressure gradient in the bad curvature region. The mirror mode with a dominant compressional magnetic field perturbation is excited when the product of plasma beta and pressure anisotropy $\left(\mathrm{P}_{\perp} / \mathrm{P}_{\|}>1\right)$ is large. In the limit that the wave frequency is smaller than the energetic trapper. article magnetic drift frequency, which is usually much smaller than the energetic trapped particle bounce frequency, the energetic trapped particles experience the bounce-averaged wave structure due to their rapid bounce motion. For modes with north-south symmetric field-aligned structure of parallel perturbed magnetic field the energetic trapped particle kinetic pressure response is finite and cancels with their fluid pressure response so that the symmetric mode is stable. Physically the energetic trapped particles precess very rapidly across the $\vec{B}$ field, and their motion becomes very rigid with respect to low frequency symmetric MHD perturbations. For antisymmetric modes the energetic trapped particle kinetic pressure response from the northern hemisphere cancels with that from the southern hemisphere in a bounce period, and the instability $\beta$ threshold of the antisymmetric mode is determined by the energetic particle fluid free energy. Pressure anisotropy with $\mathrm{P}_{\perp} / \mathrm{P}_{\|}>1$ reduces the $\beta_{\|}$threshold. The antisymmetric mode changes from a ballooning mode with dominant transverse magnetic field components at $\mathrm{P}_{\perp} / \mathrm{P}_{\|}=1$ to a hybrid ballooning-mirror type mode with comparable transverse and compressional magnetic field components near the equator as $P_{\perp} / P_{\|}$increases. With large equatorial plasma beta $\left(\beta_{\|} \geq O(1)\right)$ and pressure anisotropy $\left(P_{\perp} / P_{\|}>1\right)$ the field-aligned wave structure of antisymmetric ballooning-mirror mode resembles the multisatellite observations of a long lasting compressional Pc 5 wave event during November 14-15, 1979 [Takahashi et al., 1987]. From the AMPTE/CCE particle and magnetic field data observed during Pc 4-5 wave events we compute the ballooning-mirror instability parameters and perform a correlation study with the theoretical instability threshold. We find that compressional Pc 5 waves approximately satisfy the ballooning-mirror instability condition, and transverse Pc 4-5 waves are probably related to resonant ballooning instabilities with small pressure anisotropy. 


\section{Introduction}

Internally driven Pc 4-5 waves usually have large azimuthal mode numbers on the order of 100 and are mainly observed in the magnetosphere by satellites [Takahashi, 1988]. There are two types of internally driven ULF waves; one has a dominant compressional magnetic field component, and the other has a dominant transverse component. Compressional Pc 5 magnetic perturbations [Walker et al., 1982; Allan et al., 1982; Nägano and Araki, 1983; Misra et al., 1985; Lin and Barfield, 1985; Takahashi et al., 1985a, 1985b, 1987; Higuchi et al., 1986] with periods in the range of 150-600 seconds were believed to be driven by mirror-type instabilities [Hasegawa, 1969; Cheng and Lin, 1987]. The highly compressional magnetic perturbations are usually observed around the earth's magnetic equator near geosynchronous orbit during periods of enhanced ring current intensity and are associated with high beta, anisotropic pressure plasmas with $\mathrm{P}_{\perp}>\mathrm{P}_{\|}$, where the subscripts $\perp$ and $\|$ denote the components perpendicular and parallel to the ambient magnetic field respectively. Significant progress has been made in the multisatellite observation of a long lasting compressional Pc 5 wave event during November 14-15, 1979 [Takahashi et al., 1987; Takahashi, 1988; Takahashi et al., 1990b] which revealed the field-aligned structures of the compressional magnetic field $\delta \mathrm{B}_{\|}$to be antisymmetric with respect to the earth's magnetic equator with a full latitudinal range of about $20^{\circ}$. The smaller transverse magnetic components, radial component $\delta \mathrm{B}_{\psi}$ and azimuthal component $\delta \mathrm{B}_{\phi}$, have a symmetric parity and their polarization varies with the magnetic latitude. The wave frequencies are about one order of magnitude smaller than the shear Alfvén frequency obtained from the measured plasma density and wave structures along the ambient magnetic field at geosynchronous orbit.

The internally driven Pc 4-5 transverse waves usually have low frequency and dominant radial polarization, and they are believed to have similar (but more extended) field-aligned wave symmetry properties as the compressional Pc 5 waves [Engebretson et al., 1988; Takahashi et al., 1990a].

Linear theories of internally driven compressional Alfvén type instabilities have been presented in terms of (1) mirror instabilities due to pressure anisotropy [Hasegawa, 1969: Pokhotelov et al., 1986; Cheng and Lin, 1987], (2) drift compressional instabilities due to pressure nonuniformity [Hasegawa, 1971; $\mathrm{Ng}$ et al., 1984; Cheng and Lin, 1987], (3) trapped particle bounce and magnetic drift resonance excitation of ULF waves [Southwood, 1976; Chen and Hasegawa, 1991], and (4) the ballooning-mirror instability [Cheng and Qian, 1993]. A potential candidate for explaining internally driven transverse Alfvén type instabilities is the ballooning mode [Miura et al., 1989; Lee and Wolf, 1992; Cheng and Qian, 1993]. In general the transverse and 
compressional magnetic fields couple, and the ballooning and mirror modes are limiting cases of the general ballooning-mirror instability [Cheng and Qian, 1993].

Two coupled kinetic-MHD eigenmode equations have been derived [Cheng and Lin, 1987; Cheng, 1991, Chen and Hasegawa, 1991] to describe the coupling between the transverse and the compressional magnetic field components. The transverse magnetic field equation shows that the shear Alfvén type wave can be destabilized by the combined effect of the magnetic field curvature and the plasma pressure gradient to excite the ballooning mode. The compressional magnetic field equation indicates that the mirror mode can be destabilized by the combined effect of plasma beta and pressure anisotropy $\left(\mathrm{P}_{\perp}>\mathrm{P}_{\|}\right)$. The coupling between the compressional and transverse magnetic field components is due to finite perpendicular pressure gradient and the kinetic trapped particle perturbed pressures. The kinetic effects associated with the energetic trapped particles play an essential role in determining the stability and structure of the ballooning-mirror mode.

Cheng and Qian [1993] have performed analytical and numerical eigenmode analyses of ballooning-mirror mode that take into account the coupling between the transverse ballooning and the compressional mirror modes in anisotropic pressure plasmas along the ambient magnetic field. The ballooning-mirror mode frequency is assumed to be smaller than the energetic particle magnetic drift frequency, which is usually much smaller than the energetic trapped particle bounce frequency. Without the kinetic effects we show that the MHD mode with symmetric structure of parallel perturbed magnetic field $\delta B_{\|}$and electrostatic potential $\Phi$ along the north-south ambient magnetic field is more unstable than the antisymmetric mode. However, if energetic trapped particle kinetic effects are included, the symmetric ballooning-mirror mode is completely stabilized. On the other hand, the antisymmetric ballooning-mirror mode is only weakly influenced by energetic trapped particle kinetic effects and remains unstable. For symmetric modes, the energetic trapped particles experience the bounce-averaged wave structure due to their rapid bounce motion, and their nonadiabatic kinetic pressure response cancels with their perturbed fluid pressure response so that they don't contribute to drive the instability of the symmetric mode. Physically the energetic trapped particle stabilization of the symmetric mode results from the condition that the energetic trapped particles drift very rapidly across the $\vec{B}$ field, and their motion becomes very rigid with respect to low frequency symmetric MHD perturbations. For antisymmetric modes, the energetic trapped particle nonadiabatic kinetic pressure response from the northern hemisphere cancels with that from the southern hemisphere in a bounce period, and thus the instability $\beta$ threshold is mainly determined by the energetic particle fluid free energy. 
The field-aligned perturbed magnetic field structure of the antisymmetric mode changes from a ballooning mode with dominant transverse magnetic field component at $T_{\perp} / T_{\|}=1$ to a mixed mirror-ballooning type mode with comparable transverse and compressional magnetic field components near the equator as $T_{\perp} / T_{\|}$increases. As $T_{\perp} / T_{\|}$increases, the amplitudes of the parallel magnetic field component $\delta \mathrm{B}_{\|}$and the azimuthal component $\delta \mathrm{B}_{\phi}$ increase, and the mode also becomes more localized near the equator. At large values of $\mathrm{T}_{\perp} / \mathrm{T}_{\|}$the theoretical eigenmode structure resembles the field-aligned wave structure (all three perturbed magnetic field components) of the multisatellite (SCATHA, GOES2, GOES3, GEOS2) observations of a long lasting compressional Pc 5 wave event during November 14-15, 1979 [Takahashi et al., 1987; Takahashi, et al., 1990b] in the ring current region.

Chen and Hasegawa [1991] proposed that the perturbed compressional magnetic field with antisymmetric field-aligned structure can be excited by the drift Alfvén ballooning mirror instability that is destabilized by hot particle pressure gradient via the magnetic drift-bounce resonance. The drift Alfvén ballooning mirror instability may have lower pressure anisotropy threshold than the ballooning-mirror instability. The relationship between the ballooning-mirror mode and the drift Alfvén ballooning-mirror mode has still not yet been resolved. Even though no realistic drift Alfvén ballooning-mirror mode stability calculation has been performed, it is conjectured that the drift Alfvén ballooning mirror instability may have a slightly lower pressure anisotropy threshold than the ballooning-mirror instability. However, it is important that wave resonance with all particle species be included in the calculation before a definite answer on the stability threshold can be concluded.

Although significant progress has been made in the satellite observational data of Pc 4-5 waves and theoretical understandings of ULF waves, a close comparison of the plasma properties during wave activities between theory and observation has still not yet been performed. In the paper we present a correlation study between the theoretical ballooning-mirror instability threshold parameters and those obtained from satellite particle data during Pc 4-5 wave events. By comparing the observed plasma properties with the theoretical ballooning-mirror instability threshold one should be able to identify whether the internal excitation mechanism of observed Pc 4-5 waves is due to the ballooning-mirror instabilities.

From the AMPTE/CCE particle data and magnetic field observed during Pc 4-5 wave events we compute the instability parameters and perform a correlation study with the theoretical ballooning-mirror instability threshold parameters. It is nontrivial to compute the ballooningmirror instability parameters from the measured particle distribution along a satellite's orbit. We 
need to know how the ion distribution and the computed instability parameters associated with the ballooning-mirror instabilities change from the time interval of no wave activity to those of clear wave activity. By performing theoretical calculations based on the observed ion distributions we might be able to determine whether the wave is driven by pressure anisotropy, or pressure gradient. The results of the correlation studies indicate that the ballooning-mirror instabilities are responsible for the Pc 4-5 waves. The transverse wave is related to the resonant type ballooning mode at lower beta. The compressional wave is related to the hybrid ballooning-mirror mode excited when pressure gradient and pressure anisotropy $\left(\mathrm{P}_{\perp} / \mathrm{P}_{\|}\right)$are large.

The paper is organized as follows. In Sec. 2 we briefly review the results of a kineticMHD stability analysis of the ballooning-mirror mode [Cheng and Qian, 1993] that explains the observed wave structures in terms of predicted plasma parameters. Section 3 presents the results of the correlation study of particle data for both the transverse and the compressional wave events observed by AMPTE/CCE are presented. A summary and discussion is given in Sec. 4.

\section{Ballooning-Mirror Instabilities}

We consider a magnetospheric ring current plasma consisting of a core background component (electron and cold ions) and a hot ion component (hot proton and Oxygen) with the hot plasma density smaller than the core plasma density, $n_{h} \ll n_{c}$, and the hot plasma temperature larger than the core plasma temperature, $T_{h} \gg T_{c}$. For the ring current plasma near the geosynchronous orbit, typically the core plasma temperature is $\mathrm{T}_{\mathrm{c}} \approx 10-10^{2} \mathrm{eV}$, the hot plasma temperature is $T_{h} \approx 10^{4}-10^{5} \mathrm{eV}$, the ratio of the hot plasma density to the core plasma density is $\left(n_{h} / n_{c}\right) \approx 10^{-1}$, thus the ratio of core plasma beta to the hot plasma beta is $\left(\beta_{c} / \beta_{h}\right) \approx 10^{-2}$. Therefore, the plasma beta is dominated by the hot ion component. For such plasma its dynamic is most conveniently described by a kinetic-MHD model [Cheng, 1991].

If the plasma convection in the magnetosphere is small, the magnetospheric equilibrium can be approximated by a static MHD equilibrium with anisotropic pressure, which is described by the system of equations

$$
\begin{aligned}
\overrightarrow{\mathbf{J}} \times \overrightarrow{\mathrm{B}} & =\nabla \cdot \overrightarrow{\mathrm{P}}=\nabla \mathrm{P}_{\perp}-\nabla \bullet\left[\left(\mathrm{P}_{\perp}-\mathrm{P}_{\|}\right) \hat{\mathrm{b}} \hat{\mathrm{b}}\right] \\
\nabla \times \overrightarrow{\mathrm{B}} & =\overrightarrow{\mathbf{J}}
\end{aligned}
$$




$$
\nabla \cdot \vec{B}=0
$$

where $\hat{b}$ is a unit vector along an equilibrium magnetic field line, and $\overrightarrow{\mathbf{J}}, \overrightarrow{\mathrm{B}}$, and $\overrightarrow{\mathrm{P}}$ are the equilibrium current, magnetic field, and pressure tensor, respectively.

For an axisymmetric magnetospheric equilibrium, the magnetic field can be expressed as $\vec{B}$ $=\nabla \psi \times \nabla \phi$ and the equilibrium solution has been studied numerically by Cheng [1992]. For a dipole field the magnetic flux function given by $\psi=-M \sin ^{2} \theta / r$, where $r$ and $\theta$ are the radius and polar angle in the spherical coordinate system $(r, \theta, \phi)$, and $M$ is the dipole moment. The components of the dipole magnetic field are given by $B_{r}=-2 M \cos \theta / r^{3}$, and $B_{\theta}=-M \sin \theta /$ $r^{3}$. For a collisionless plasma the particle energy $\left(\varepsilon=v^{2} / 2\right)$ and the adiabatic invariants, magnetic moment $\left(\mu=v_{\perp}{ }^{2} / 2 B\right)$ and the longitudinal invariant $\left(J_{\|}=\int d s v_{\|}\right)$, are constant during the drift motions, where $v_{\|}$and $v_{\perp}$ are the components of the velocity parallel and perpendicular to $\vec{B}$, respectively. The guiding center equilibrium particle distribution function must have the form $F=$ $\mathrm{F}\left(\varepsilon, \mu, J_{\|}\right)$. In general, $\mathrm{J}_{\|}=\mathrm{J}_{\|}(\varepsilon, \mu, \psi, \alpha)$ and $\mathrm{F}=\mathrm{F}(\varepsilon, \mu, \psi, \alpha)$. If all particles on each field line share the same drift surface, where $\psi$ labels the drift surface, then $J_{\|}=J_{\|}(\varepsilon, \mu, \psi)$ and $F=$ $\mathrm{F}(\varepsilon, \mu, \psi)$. The guiding-center particle distributions $\mathrm{F}(\varepsilon, \mu, \psi)$ can be either prescribed by an analytical form or obtained from the satellite measurements of the particle flux. The equilibrium parallel and perpendicular pressures are defined by

$$
\left.\left(\begin{array}{l}
P_{\|} \\
P_{\perp}
\end{array}\right)=\sum_{j, \sigma_{\|}} 2 \pi m_{j} \int_{0}^{\alpha} d \varepsilon \int_{0}^{\varepsilon / B} d \mu\left|B F_{j} /\right| v_{\|} \mid\right]\left(\begin{array}{c}
2(\varepsilon-\mu B) \\
\mu B
\end{array}\right),
$$

where the summation is over the particle species $\mathrm{j}$ and $\sigma_{\|}$which represents the direction of particle velocity parallel to $\vec{B}$, and $m_{j}$ is the particle mass. The parallel velocity $v_{\|}$has the form

$$
\left.v_{\|}=\sigma_{\|} \sqrt{2} \overline{\varepsilon-\mu B}\right) \text {. }
$$

By inspection $\mathrm{P}_{\perp}$ and $\mathrm{P}_{\|}$are functions of $\psi$ and $\mathrm{B}$ only. It is convenient to introduce the functions

$$
\sigma \equiv 1+\left(\mathrm{P}_{\perp}-\mathrm{P}_{\|}\right) / \mathrm{B}^{2}
$$

and

$$
\tau \equiv 1+(1 / \mathrm{B})\left(\partial \mathrm{P}_{\perp} / \partial \mathrm{B}\right)_{\psi}
$$


The momentum balance equation parallel to the equilibrium magnetic field is given by

$$
\vec{B} \cdot \nabla P_{\|}=\left(P_{\|}-P_{\perp}\right) \hat{b} \cdot \nabla B
$$

Making use of Eqs. (6)-(8), the momentum balance equation perpendicular to the magnetic field is given by

$$
\tau \nabla_{\perp}\left(B^{2} / 2\right)+\tilde{\nabla}_{\perp} P_{\perp}=\vec{\kappa} \sigma B^{2}
$$

where $\widetilde{\nabla}=\nabla-\nabla \mathrm{B}(\partial / \partial \mathrm{B})_{\psi}, \overrightarrow{\mathrm{K}}=\hat{\mathrm{b}} \bullet \nabla \hat{\mathrm{b}}$ is the magnetic field curvature. Note that the parallel momentum balance equation, Eq. (8), is automatically satisfied if the particle distribution $F(\varepsilon, \mu, \psi)$ is used to compute $\mathrm{P}_{\perp}$ and $\mathrm{P}_{\|}$. From Eq. (8), $\sigma$ can be expressed as $\sigma=1-(1 / \mathrm{B})\left(\partial \mathrm{P}_{\|} / \partial \mathrm{B}\right)_{\psi}$.

The linearized kinetic-MHD eigenmode equations for the low-frequency transverse and compressional Alfvén waves had been derived [Cheng and Lin, 1987; Cheng, 1991]. By assuming that the perpendicular wavelength is shorter than the parallel wavelength which is in turn shorter than the equilibrium scale length and the particle gyroradius is small compared to the perpendicular wavelength for all particle species, the perturbed guiding center particle distribution $\delta \mathrm{f}$ is determined from the drift kinetic equation. In the limit of zero parallel electric field the perturbed transverse magnetic field is related to the electrostatic potential $\Phi$ by $\delta \overrightarrow{\mathrm{B}}_{\perp} \approx \nabla \times[\overrightarrow{\mathrm{B}}(\overrightarrow{\mathrm{B}} \bullet \nabla$ $\left.\Phi / \omega B^{2}\right)$ ], where $\omega$ is the eigenmode frequency of the perturbed quantities which have a temporal dependence of $\exp (-\mathrm{i} \omega \mathrm{t})$. We consider a WKB wave form perpendicular to the ambient magnetic field so that $\nabla_{\perp}$ operates only on the perturbed quantities and $\nabla_{\perp}=i \vec{k}_{\perp}$. The eigenmode equations for $\widehat{\Phi}(=\Phi / \omega)$ and $\delta \mathrm{B}_{\|}$can be obtained from Eqs. (40), (41), and (45) in the paper by Cheng [1991] and are given by

$$
\begin{aligned}
\overrightarrow{\mathrm{B}} \bullet \nabla\left(\frac{\sigma \mathrm{k}_{\perp}^{2}}{\mathrm{~B}^{2}} \overrightarrow{\mathrm{B}} \bullet \nabla \hat{\Phi}\right)+\frac{\rho \mathrm{k}_{\perp}^{2} \omega^{2}}{\mathrm{~B}^{2}} \hat{\Phi} \\
\quad+\frac{(\overrightarrow{\mathrm{B}} \times \overrightarrow{\mathrm{K}})}{\mathrm{B}^{2}} \cdot \overrightarrow{\mathrm{k}}_{\perp}\left[\frac{\overrightarrow{\mathrm{B}} \times \tilde{\nabla}_{\|}}{\mathrm{B}^{2}} \cdot \overrightarrow{\mathrm{k}}_{\perp} \hat{\Phi}+\sigma \mathrm{B} \delta \mathrm{B}_{\|}-\delta \hat{\mathrm{p}}_{\|}\right]=0,
\end{aligned}
$$

and 


$$
\begin{aligned}
\left.\overrightarrow{\mathrm{B}} \cdot \nabla \mid \frac{\sigma}{\mathrm{B}^{2}} \overrightarrow{\mathrm{B}} \cdot \nabla \delta \mathrm{B}_{\|}\right\rfloor & +\frac{\rho \omega^{2}}{\mathrm{~B}^{2}} \delta \mathrm{B}_{\|} \\
& -\mathrm{k}_{\perp}^{2}\left[\tau \delta \mathrm{B}_{\|}-\overrightarrow{\mathrm{k}}_{\perp} \cdot \frac{\overrightarrow{\mathrm{B}} \times \widetilde{\nabla P}_{\perp}}{\mathrm{B}^{3}} \hat{\Phi}+\frac{\delta \hat{\mathrm{p}}_{\perp}}{\mathrm{B}}\right]=0,
\end{aligned}
$$

where $\rho$ is the mass density, $\tilde{\nabla}=\nabla-\nabla \mathrm{B}(\partial / \partial \mathrm{B})_{\psi}$. The kinetic effect due to trapped particles is included in the nonadiabatic perturbed pressures $\delta \hat{\mathrm{p}}_{\perp}$ and $\delta \widehat{\mathrm{p}}_{\|}$which are given by

$$
\left(\begin{array}{c}
\delta \hat{\mathrm{p}}_{\|} \\
\delta \hat{\mathrm{p}}_{\perp}
\end{array}\right)=\sum_{j} \mathrm{M} \int \mathrm{d}^{3} v \hat{\mathrm{g}}\left(\begin{array}{c}
2(\varepsilon-\mu \mathrm{B}) \\
\mu \mathrm{B}
\end{array}\right)
$$

and in the absence of parallel electric field the drift kinetic equation for the linearized nonadiabatic particle distribution function $\widehat{\mathrm{g}}$ is given by

$$
\left[\frac{\partial}{\partial t}+\left(\vec{v}_{\|}+\vec{v}_{d}\right) \cdot \nabla\right] \widehat{g}=\left[\frac{e}{M} \frac{\partial F}{\partial \varepsilon} \frac{\partial}{\partial t}-\frac{\vec{B} \times \nabla F}{B^{2}} \cdot \nabla\right] Y,
$$

where $Y=i \vec{v}_{d} \bullet \nabla \Phi / \omega-M \mu \delta B_{\|} / e, \vec{v}_{d}=\left(M \vec{B} / e B^{2}\right) \times\left[\nabla(\mu B)+\vec{\kappa}_{\|_{\|}}{ }^{2}\right]$ is the particle magnetic drift velocity in the equilibrium magnetic field, and $\vec{K}=(\vec{B} / B) \bullet \nabla(\vec{B} / B)$ is the equilibrium magnetic field curvature. In terms of a Fourier series representation the linearized nonadiabatic particle distribution function $\hat{g}$ has been derived for a magnetospheric geometry in the paper by Cheng [1991] (see Sec. 4 and Appendix C) and will not be repeated here..

Equations (10) and (11) describe the coupling between the compressional and transverse perturbed magnetic field due to finite magnetic field curvature, perpendicular pressure gradient, and the nonadiabatic trapped particle kinetic pressure responses $\delta \hat{\mathrm{p}}_{\perp}$ and $\delta \hat{\mathrm{p}}_{\|}$. Eq. (10) describes the transverse shear Alfvén type wave that can be destabilized by pressure gradient in a bad magnetic field curvature with $(\vec{\kappa} \bullet \nabla \psi)(\nabla \mathrm{P} \bullet \nabla \psi)>0$ to excite ballooning modes in a high $\beta(=\mathrm{O}(1))$ nonuniform plasma. In Eq. (10) the first term represents the stabilizing field line bending effect for $\sigma>0$, the second term represents the core plasma inertia, the third term represents the destabilization effect of pressure gradient in a bad magnetic field curvature, the $\delta B_{\|}$term represents the coupling to the compressional Alfvén type wave, and the $\delta \hat{\mathrm{p}}_{\|}$term represents the nonadiabatic (kinetic) hot trapped particle parallel perturbed pressure. Equation (11) indicates that the 
compressional Alfvén type waves can be destabilized by the the combined effect of plasma beta and pressure anisotropy to excite mirror modes. The mirror instability free energy is represented by the parameter $\tau$ defined in Eq.(7). In Eq. (11) the first term represents a stabilizing field line propagating effect for $\sigma>0$, the second term represents the core plasma inertia, the third term represents the pressure anisotropy effect on the perpendicular propagation of compressional wave, the $\Phi$ term represents the coupling to the shear Alfvén type wave, and the $\delta \hat{\mathrm{p}}_{\perp}$ term represents the nonadiabatic (kinetic) hot trapped particle perpendicular perturbed pressure. If $\tau<0$ mirror mode can be unstable with perturbation localized around the region where $\tau<0$. Eqs. (10) and (11) are similar to the previously presented eigenmode equations (Berk et al., 1983; Cheng and Lin, 1987; Chen and Hasegawa, 1991]. However, we note that the first two terms of Eq.(11) were neglected in Eq. (21) of Chen and Hasegawa [1991]; the first term was neglected because they assumed that $\mathrm{k}_{\|}{ }^{2}<<\mathrm{k}_{\perp}{ }^{2}$, and the second term was neglected because they employed the low frequency gyrokinetic equations that ignore compressional Alfvén type waves. In our studies, we retained these two terms in Eq.(11) to study the coupling between the ballooning and mirror modes even in the limit that $\tau$ goes to zero.

The stability of the antisymmetric ballooning mirror mode is usually expressed in terms of parallel plasma beta $\beta_{\|}$and temperature ratio $T_{\perp} / T_{\|}$at equator. The stability property can also be expressed in terms of $\alpha_{p}$ and $\tau$, where the new dimensionless parameter $\alpha_{p}$ is defined to describe the ballooning instability free energy and is given by

$$
\alpha_{p}=\left(\frac{\vec{k}_{\perp} \times \vec{B} \bullet \vec{k}}{k_{\perp} B}\right) \frac{\vec{k}_{\perp} \times \vec{B}}{k_{\perp} B^{3}} \cdot\left[\left(\frac{\sigma}{\tau}\right) \tilde{\nabla} P_{\perp}+\widetilde{\nabla} P_{\|}\right] L^{2},
$$

where $\mathrm{L}$ is the equatorial $\mathrm{L}$-shell distance. If $\alpha_{\mathrm{p}}>0$ the ballooning instability can occur with perturbation localized around the region where $\alpha_{p}>0$.

By totally neglecting kinetic trapped particle pressures, the eigenmode equations are essentially MHD equations with anisotropic pressure along the ambient magnetic field line, and the MHD ballooning modes with symmetric $\Phi$ and $\delta \mathrm{B}_{\|}$structures along the north-south equilibrium magnetic field lines have the lowest beta stability threshold. The antisymmetric MHD modes have higher beta stability threshold. It has been shown [Cheng and Qian, 1993] that when the kinetic contribution from trapped particle pressures is included in the analysis, the symmetric ballooning modes are stabilized by the energetic trapped particle compressional effect in the absence of waveparticle resonance. On the other hand, the kinetic trapped particle effects on the stability of the 
antisymmetric mode are vanishingly small. Therefore, the antisymmetric modes have the lowest critical beta threshold for balloonirig-mirror instability. For small pressure anisotropy the antisymmetric MHD mode is a pure ballooning mode. But, for higher pressure anisotropy with simple mirror threshold ( $\tau=0$ ) exceeded at equator, the antisymmetric MHD mode becomes a hybrid mirror-ballooning mode with strong coupling between the transverse and the parallel magnetic field components.

Figure 1(a) shows the marginal stability boundaries of the antisymmetric ballooning-mirror mode in the equatorial $\left(\beta_{\|}, \mathrm{T}_{\perp} / \mathrm{T}_{\|}\right)$space for $\partial \ln \mathrm{P} / \partial \ln \mathrm{L}=-100,-50,-25,-10,-5,-2.5$, and -1 , respectively, where $P$ is the pressure in the isotropic limit. The fixed parameters are $L=6.6 R_{E}$ and azimuthal mode number $m=50$. Also shown in Fig. 1(a) is the equatorial $\tau=0$ curve above which the mirror instability criterion is satisfied at equator. The pressure anisotropy is destabilizing and can lower the critical beta for the ballooning-mirror mode. For $T_{\perp} / T_{\|}>1.6$ ( with $\partial \ln \mathrm{P} / \partial \ln \mathrm{L}=-5)$ the critical beta is larger than the beta values that satisfy $\tau=0$. For equatorial $\left(\beta_{\|}, T_{\perp} / T_{\|}\right)$values that are above the $\tau=0$ curve, the mode has a dominant mirror mode characteristics. The marginal stability boundaries in the equatorial $\left(\beta_{\|}, T_{\perp} / T_{\|}\right)$space are very sensitive to the pressure gradient.

The marginal stability boundaries of the ballooning-mirror mode can also be given in terms of the equatorial values of $\alpha_{p}$ and $\tau$. The corresponding stability boundaries are also shown in Fig. 1(b) in the equatorial $\left(\alpha_{p}, \tau\right)$ space. Note that for $\tau<0$, the $\alpha_{p}$ threshold is also negative, and we have plotted absolute value of $\alpha_{\mathrm{p}}$ in Fig. 1(b) for $\tau<0$. We note that the marginal stability boundary curves in $\left(\tau, \alpha_{p}\right)$ space is very close to each other even for $10>|\partial \ln P / \partial \ln L|>0.1$. This probably holds even for different type of particle velocity distributions. This property is useful for comparing theoretical calculations with satellite observations where information on $\partial \ln \mathrm{P} / \partial \ln \mathrm{L}$ and particle velocity distribution is either unavailable or can not be obtained accurately. Therefore, we will employ $\tau$ and $\alpha_{p}$ parameters to compare theoretical ballooning-mirror instability thresholds with those obtained from satellite observed particle data where information on $\partial \ln P / \partial \ln L$ is either unavailable or can not be obtained accurately.

The field-aligned structure of the perturbed magnetic field of the antisymmetric mode changes from a pure ballooning mode with dominant transverse magnetic field component at $T_{\perp} / T_{\|}$ $\approx 1$ to a mixed mirror-ballooning type mode with comparable transverse and compressional magnetic field components near the equator as $T_{\perp} / T_{\|}$increase (or $\tau$ decreases). Figures $2(a)$ - (d) show the field-aligned perturbed magnetic field structure of the marginally stable antisymmetric mode along a marginal stability curve in the $\left(\beta_{\|}, \mathrm{T}_{\perp} / \mathrm{T}_{\|}\right)$space as shown in Fig. 8(a) with equatorial 
values of (a) $T_{\perp} / T_{\|}=1, \beta_{\|}=1.93(\tau=1)$, (b) $T_{\perp} / T_{\|}=1.3, \beta_{\|}=1.489$ ( $\tau=0.419$ ), (c) $T_{\perp} / T_{\|}=$ 1.6, $\beta_{\|}=0.987(\tau=0.052)$, and $(\mathrm{d}) \cdot \mathrm{T}_{\perp} / \mathrm{T}_{\|}=2, \beta_{\|}=0.575(\tau=-0.149)$. The fixed parameters are $\mathrm{L}=6.6$, azimuthal mode number $\mathrm{m}=50$, and $\partial \ln \mathrm{P} / \partial \ln \mathrm{L}=-5$. For positive values of $\tau$, the local dispersion indicates a ballooning mode along the field line. As $\tau$ decreases, the amplitudes of the parallel magnetic field component $\delta \mathrm{B}_{\|}$and the azimuthal component $\delta \mathrm{B}_{\phi}$ increase, and the mode also becomes more localized near the equator. At negative or small positive values of $\tau$ the theoretical ballooning-mirror mode structure resembles the field-aligned wave structures (all three perturbed magnetic field components) of the multisatellite (SCATHA, GOES2, GOES3, GEOS2) observations of a long lasting compressional Pc 5 wave event during November 14-15, 1979 [Takahashi et al., 1987; Takahashi, et al., 1990b]. This strongly suggests that this particular multisatellite observation may be related to the marginally stable ballooning-mirror mode with $\tau$ value close to zero.

\section{Correlation Studies with Particle Data and Wave Events Observed by AMPTE/CCE}

The AMPTE/CCE spacecraft was launched in August 1984 and was operated until early 1989. It had a perigee of $1.2 \mathrm{R}_{\mathrm{E}}$ geocentric, an apogee of $8.8 \mathrm{R}_{\mathrm{E}}$, an inclination of $5^{\circ}$, and an approximate orbital period of 16 hours. The satellite was spin stabilized with a spin axis maintained within $30^{\circ}$ of the sun-Earth line. The spin period was approximately $5.9 \mathrm{sec}$.

The CCE magnetometer sampled 8 vectors/sec [Potemra et al., 1985]. Six-second median magnetic field record from CCE was plotted on michrofiche for the time interval of August, 1984 to December, 1986 and were scanned for magnetic pulsations in the Pc 4-5 band (period $=45-600)$ s). We only selected events occurring near the dipole equator, because for these events it is easy to distinguish between waves of symmetric and antisymmetric field-aligned structures and also because near the equator the particle detector almost completely measured the particles trapped in the magnetic field. Four transverse wave events and six compressional wave events are selected and listed in Table 1. The distinction between compressional and transverse modes are made based on the relative magnitude of the field-aligned to the transverse components.

For each event in Table 1 we have analyzed particle data acquired with the ion chargeenergy-mass spectrometer (CHEM). The CHEM instrument measures the energy and pitch angle distribution of all major ions in the magnetosphere [Gloeckler et al., 1985]. The energy coverage is from $0.3 \mathrm{keV} / \mathrm{charge}$ to $300 \mathrm{keV} / \mathrm{ch}$ arge. The pitch angle information is obtained by dividing the 
scan plane ( = spin equator of the satellite) into 32 sectors. Because the satellite spin axis was nearly perpendicular to the Earth's dipole field, the pitch angle coverage was nearly complete. A complete energy-pitch angle scan for a species is obtained at $\sim 3 \mathrm{~min}$ intervals, and the particle pressure can be determined rather accurately in spite of the limited energy coverage [Lui et al., 1987; Lui and Hamilton, 1992]. CHEM measures particle flux of four major ions species $\mathrm{H}^{+}$, $\mathrm{He}^{+}, \mathrm{He}^{++}$, and $\mathrm{O}^{+}$. In general the contribution of $\mathrm{He}^{+}$and $\mathrm{He}^{++}$to the plasma beta is very small and can be neglected. The contribution of $\mathrm{O}^{+}$to the plasma beta can be as large as $30 \%$ of the total plasma beta during storm time and can be important in computing the parameters $\tau$ and $\alpha_{p}$. However, due to low counting statistics the pitch angle distribution of $\mathrm{O}^{+}$is not well determined and is not used in computing $\tau$ and $\alpha_{p}$ [Cheng et al., 1994]. On the other hand, the pitch angle and energy distribution of $\mathrm{H}^{+}$is relatively well determined and were used to compute the parameters $\tau$ and $\alpha_{\mathrm{p}}$. In the present analysis we will neglect the $\mathrm{O}^{+}$contribution and retain only $\mathrm{H}^{+}$ contribution. We will average the sectored $\mathrm{H}^{+}$ion data over bins of $0.2 \mathrm{R}_{\mathrm{E}}$ width in the $\mathrm{L}$ shell distance.

The transverse wave events are typical radially polarized Pc 4 magnetic pulsations observed on the dayside during geomagnetically quiet times. During the period the transverse waves are observed, the proton betas are enhanced, but remain relatively small with $\beta_{\|} \approx 0.05-0.25, \beta_{\perp} \approx$ $0.1-0.4$, and the pressure anisotropy parameter $\tau$ is close to unity with $\tau>0.6$. The compressional wave events are selected from the 23 compressional Pc 5 wave events studied by Takahashi et al. 11987; 1990b]. As Takahashi et al. [1987] noted, very near the equator the transverse and the compressional components exhibit comparable amplitude with an occasional second harmonic in the compressional component. In comparison with the transverse wave events the period of the compressional waves is longer and their amplitude relative to the ambient field $\delta \mathrm{B} / \mathrm{B}$ is larger. During the period that there is no wave activity the plasma betas are usually less than one. But, when the compressional waves are observed, the plasma betas are greatly increased and become larger than one with $\beta_{\|} \approx 1-5$, and the pressure anisotropy parameter $\tau$ is somewhat small and can become negative with $\tau<0.6\left(\mathrm{P}_{\perp} / \mathrm{P}_{\|} \approx 1.1-1.5\right)$.

In Fig. 3 we plotted the values of $\tau$ and $\alpha_{p}$ computed from the AMPTE/CCE particle data separately during (a) the transverse wave events and (b) the compressional wave events listed in Table 1. Also plotted in Fig. 3 are the marginal stability boundary curves of the antisymmetric $\delta B_{\|}$ ballooning-mirror instability numerically computed for a local bi-Maxwellian velocity distribution with the pressure gradient in the range of $100>|\partial \ln \mathrm{P} / \partial \operatorname{lnL}|>1$. The observation points in the $\left(\tau, \alpha_{p}\right)$ space were calculated for the period listed as "Data Interval" in Table 1. The symbol " $)$ " represents compressional wave events, and the symbol " + "represents the transverse wave 
activities. When there is no wave activity the values of $\tau$ and $\alpha_{p}$ tend to be well below the ballooning-mirror thresholds. The transverse and the compressional wave events clearly occupy different domains in the $\left(\tau, \alpha_{p}\right)$ space. For the compressional wave events the values of $\tau$ and $\alpha_{p}$ are either near or above the theoretical marginal stability boundary curves of the antisymmetric $\delta B_{\|}$ ballooning-mirror modes. The compressional wave events tend to occur when $\tau \leq 0.6$ and $\mathrm{O}(10)$ $\leq \alpha_{p} \leq \mathrm{O}\left(10^{3}\right)$. Thus, the compressional waves are probably related to hybrid ballooning-mirror modes excited when the pressure gradient, the plasma beta, and the pressure anisotropy $\left(\mathrm{P}_{\perp} / \mathrm{P}_{\|}\right)$are large. The transverse wave events tend to occur when $\tau$ is close to 1 and $0<\alpha_{p} \leq O(10)$ which are about one order of magnitude below the theoretical ballooning-mirror instability thresholds for antisymmetric $\delta \mathrm{B}_{\|}$mode. Thus, the transverse waves are probably related to resonant type ballooning instabilities such as the drift Alfvén ballooning mirror instability [Chen and Hasegawa, 1991] that can be excited at lower plasma beta and pressure anisotropy.

\section{Summary and Discussion}

In the paper we have presented a kinetic-MHD eigenmode stability study of the ballooningmirror mode for anisotropic pressure plasmas in a dipole magnetic field. The eigenmode equations take into account the coupling of the transverse and compressional components of the perturbed magnetic field. The symmetric modes are stabilized by the energetic trapped particle kinetic effect in the absence of wave-particle resonance. The stabilization results from the fact that the hot trapped particles precess very rapidly across the $\vec{B}$ field, their motion becomes very rigid with respect to low frequency MHD perturbations with symmetric structure of parallel perturbed magnetic field $\delta B_{\|}$and electrostatic potential $\Phi$ along the north-south ambient magnetic field, and thus they do not contribute to the destabilizing pressure gradient to drive ballooning instability. On the other hand, the energetic trapped particle's rapid bounce motion tends to average out the nonadiabatic pressure contribution associated with the antisymmetric wave structure in a bounce period. Therefore, the antisymmetric mode is only weakly influenced by energetic trapped particle kinetic effects and has the lowest critical beta threshold mainly determined by MHD theory.

Based on a local bi-Maxwellian velocity distribution, the stability of the ballooning-mirror mode is numerically computed and is expressed in terms of parallel plasma beta $\beta_{\|}$and temperature ratio $T_{\perp} / T_{\|}$at equator. The field-aligned perturbed magnetic field structure of the antisymmetric mode changes from a ballooning mode with dominant transverse magnetic field component at $\mathrm{T}_{\perp} / \mathrm{T}_{\|}=1(\tau=1)$ to a hybrid mirror-ballooning mode with comparable transverse and compressional magnetic field components near the equator as $T_{\perp} / T_{\|}$increases (i.e., $\tau$ decreases). 
As $\tau$ decreases, the amplitudes of the parallel magnetic field component $\delta \mathrm{B}_{\|}$and the azimuthal component $\delta \mathrm{B}_{\phi}$ increase, and the mode also becomes more localized near the equator. In fact, at negative or small positive values of $\tau$ the theoretical eigenmode structure resembles the fieldaligned wave structure (all three perturbed magnetic field components) of the multisatellite (SCATHA, GOES2, GOES3, GEOS2) observations of a long lasting compressional Pc 5 wave event during November 14-15, 1979 [Takahashi et al., 1987; Takahashi, et al., 1990b]. This suggests that this particular multisatellite observation may be related to the marginally stable ballooning-mirror mode with $\tau$ value close to zero.

From the AMPTE/CCE particle and magnetic field data observed during Pc 4-5 wave events we have computed the instability parameters and performed a correlation study with the theoretical ballooning-mirror instability threshold parameters in the $\left(\tau, \alpha_{p}\right)$ space so that close comparison with the theoretical instability thresholds can be made. The particle data were acquired with the ion charge-energy-mass spectrometer (CHEM). The CHEM instrument measures the energy and pitch angle distribution of all major ions in the magnetosphere. The energy coverage is from $1 \mathrm{keV} /$ charge to $300 \mathrm{keV} /$ charge (fluxes below $1 \mathrm{keV}$ are unreliable), and the pitch angle coverage was nearly complete. The transverse and the compressional wave events are found to occupy different domains in the $\left(\tau, \alpha_{p}\right)$ space. The transverse wave events tend to occur when $\tau$ is close to 1 and $0<\alpha_{p} \leq O(10)$. The compressional wave events tend to occur when $\tau \leq 0.5$ and $\alpha_{p}$ $\geq \mathrm{O}\left(10^{2}\right)$. The results of the correlation studies are consistent with the theory of ballooning-mirror instabilities. Therefore, the transverse wave is related to the ballooning mode at lower beta. The compressional wave is relateci to a mixed ballooning-mirror mode excited when pressure gradient and pressure anisotropy $\left(\mathrm{P}_{\perp} / \mathrm{P}_{\|}\right)$are large.

It is perhaps not surprising that the internally driven Pc 4-5 waves are related to the MHD type ballooning-mirror instabilities because these Pc 4-5 waves are usually not of bursty type. A resonant type instability tends to have bursty behavior in time because as soon as the resonant particles release their energy and are scattered out of resonance with the wave, the wave will be damped by other resonant particles that takes energy from the wave. On the other hand, an MHD type instability is destabilized by the fluid free energy and is weakly resonating with all particle species. After the fluid free energy is released, the wave become nearly marginally stable due to very weak resonant damping and can survive for a very long period of time. We also note that long period compressional Pc 5 waves are usually observed during recovery phase of a substorm. The substorm recovery time is roughly the time it takes to stabilize the compressional Pc 5 waves by transporting or dissipating excess hot ring ion pressure out of the ring current region. 
One of the future efforts will be to explore new satellite data of ring particle distribution as input to the self-consistent equilibrium calculation presented. One possibility is to gather data from all ion instruments on board AMPTE/CCE which cover an energy ranch of $1-600 \mathrm{keV}$ and a nearly full range of pitch angle.

\section{Acknowledgments}

This work was supported by NSF Grant No. ATM-9217859 and DoE Contract No. DEAC02-76-CHO3073 at Princeton University, and in part by NSF Grant No. ATM-9114316 at The Johns Hopkins University. 


\section{References}

Allan, W., E. M. Poulter, and E. Nielsen, STARE observations of a Pc 5 pulsation with large azimuthal wave number, J. Geophys. Res., 87, 6163, 1982.

Chen, L., and A. Hasegawa, Kinetic theory of geomagnetic pulsations 1. internal excitations by energetic particles, J. Geophys. Res., 96, 1503, 1991.

Cheng, C. Z. and C. S. Lin, Eigenmode analysis of compressional waves in the magnetosphere, Geophys. Res. Lett., 14, 884, 1987.

Cheng, C. Z., A kinetic-MHD model for low frequency phenomena, J. Geophys. Res., 96, $21159,1991$.

Cheng, C. Z., Magnetospheric equilitıium with anisotropic pressure, J. Geophys. Res., 97. 1497, 1992.

Cheng, C. Z., and Q. Qian, Theory of ballooning-mirror instabilities for anisotropic pressure plasmas in the magnetosphere, Princeton Plasma Physics Laboratory Report, PPPL-2935. submitted to J. Geophys. Res., 1993.

Cheng, C. Z., K. Takahashi, and A. T. Y. Lui, Correlation between ballooning-mirror instabilities and ULF waves events, to be submitted to J. Geophys. Res., 1994.

Engebretson, M. J., L. J. Zanetti, T. A. Potemra, D. M. Klumpar, R. J. Strangeway, and M. H. Acuna, Observations of intense ULF pulsation activity near the geomagnetic equator during quiet times, J. Geophys. Res., 93, 12795, 1988.

Gloeckler, G., F. M. Ipavich, W. Studemann, B. Wilken, D. C. Hamilton, G. Kremser, D. Hovestadt, F. Gliem, R. A. Lundgren, W. Riek, E. O. Tums, J. C. Cain, L. S. Masung, W. Weiss, and P. Winterhof, The charge-energy-mass spectrometer for $0.3-300 \mathrm{keV} / \mathrm{e}$ ions on the AMPTE CCE, IEEE Trans. Geosci. Remote Sen., GE-23, 234-240, 1985.

Hasegawa, A., Drift mirror instabilities in the magnetosphere, Phys. Fluids, 12, 2642, 1969.

Hasegawa, A., Drift wave instabilities of a compressional mode in a high b plasma, Phys. Rev. Lett., 27, 11, 1971.

Higuchi, T., S. Kokubun, and S. Ohtani, Harmonic structure of compressional Pc 5 pulsations at synchronous orbit, Geophys. Res. Lett., 13, 1101, 1986.

Lanzerotti, L. J., A. Hasegawa, and C. G. MacLennan, Drift mirror instability in the magnetosphere: Particle and field oscillations and electron heating, J. Geophys. Res., 74. $5565,1969$.

Lee, D.-Y., and R. A. Wolf, Is the Earth's magnetotail balloon unstable?, J. Geophys. Res., 97. 19251, 1992. 
Lin, C. S., and J. N. Barfield, Azimuthal propagation of storm time PC 5 waves observed simultaneously by geostationary satellites GOES2 and GOES3, J. Geophys. Res., 90. $11075,1985$.

Lui, A. T. Y., R. W. McEntire, and S. M. Krimigis, Evolution of the ring current during two geomagnetic storms, J. Geophys. Res., 92, 7459, 1987.

Lui, A. T. Y., and D. C. Hamilton, Radial profiles of quiet time magnetospheric parameters, J. Geophys. Res., 97, 19,325, 1992.

Misra, K. D., E. Nielsen, and L. Luhr, STARE and SABRE observations of a Pc 5 with large azimuthal wave number during a storm period, J. Geophys. Res., 90, 1()923, 1985.

Miura, A., S. Ohtani, and T. Tamao, Ballooning instabilities and structure of diamagnetic hydromagnetic waves in a model magnetosphere, J. Geophys. Res., 94, 15231, 1989.

Nagano, H., and T. Araki, Long-duration Pc 5 pulsations observed by geostationary satellites. Geophys. Res. Lett., 10, 908, 1983.

Ng, P. H., V. L. Patel, and S. Chen, Drift compressional instability in the magnetosphere, J. Geophys. Res., 89, 10763, 1984.

Pokhotelov, O. A., V. A. Pilipenko, Yu. M. Nezlina, J. Woch, G. Kremser, A. Korth, and E. Amata, Excitation of high-b plasma irstabilities at the geostationary orbit: theory and observations, Planet. Space Sci., 34, 695, 1986.

Potemra, T. A., L. J. Zanetti, and M. H. Acuna. The AMPTE CCE magnetic field experiment. IEEE Trans. Geosci. Remote Sen., GE-23, 246-249, 1985.

Southwood, D. J., A general approach to low-frequency instability in the ring current plasma. J Geophys. Res., 81, 334(), 1976.

Spence, H. E., M. G. Kivelson, and R. J. Walker, Magnetospheric plasma pressures in the midnight meridian: Observations from 2.5 to 35 RE, J. Geophys. Res., 94, 5264, 1989.

Takahashi, K., P. R. Higbie, and D. N. Baker, Azimuthal propagation and frequency characteristic of compressional Pc 5 waves observed at geostationary orbit, J. Geophys. Res., 90, 1473, 1985a.

Takahashi, K., C. T. Russell, and R. R. Anderson, ISEE 1 and 2 observation of the spatial structure of a compressional Pc 5 wave, Geophys. Res. Lett., 12, 613, 1985 b.

Takahashi, K., J. F. Fennell, E. Amata, and P. R. Higbie, Field-aligned structure of the storm time Pc 5 waves of November 14-15, 1979, J. Geophys. Res., 92, 5857, 1987.

Takahashi, K., Multisatellite studies of ULF waves, Advance in Space Res., 8, 427,1988.

Takahashi, K., R. W. McEntire, A. T. Y. Lui, and T. A. Potemra, Ion flux oscillations associated with a radially polarized transverse Pc 5 magnetic pulsation, J. Geophys. Res., 95, 3717. 1990a. 
Takahashi, K., C. Z. Cheng, R. W. McEntire, and L. M. Kistler, Observation and theory of Pc 5 waves with harmonically related transverse and compressional components, J. Geophys. Res., 95, 977, 1990b.

Walker, A. D. M., R. A. Greenwald, A. Korth, and G. Kremser, STARE and GEOS2 observations of a storm time Pc 5 ULF pulsation, J. Geophys. Res., 87, 9135, 1982. 
Table 1

List of Selected Wave Events

\begin{tabular}{lcccccccc}
\hline Year & Day & \multicolumn{2}{l}{$\begin{array}{l}\text { Data Interval, } \\
\text { UT }\end{array}$} & & \multicolumn{2}{l}{$\begin{array}{l}\text { MLAT Range, } \\
\text { degrees }\end{array}$} & $\begin{array}{l}\text { Event Interval, } \\
\text { UT }\end{array}$ & Wave Mode \\
\hline 84 & 245 & 0900 & 1100 & 1 & 3 & 0930 & 1030 & Transverse \\
84 & 251 & 2130 & 2330 & -1 & 1 & 2150 & 2300 & Transverse \\
85 & 324 & 2140 & 2340 & 0 & 2 & 2200 & 2310 & Transverse \\
86 & 339 & 1510 & 1740 & -5 & -1 & 1650 & 1740 & Transverse \\
& & & & & & & & \\
85 & 256 & 1700 & 1900 & -4 & 1 & 1740 & 1830 & Compressional \\
85 & 259 & 0100 & 0500 & -3 & 2 & 0300 & 0400 & Compressional \\
85 & 263 & 0100 & 0400 & 0 & 4 & 0130 & 0250 & Compressional \\
85 & 278 & 0000 & 0500 & -3 & 4 & 0300 & 0500 & Compressional \\
85 & 284 & 0320 & 0610 & 0 & 3 & 0350 & 0540 & Compressional \\
85 & 286 & 1500 & 1900 & -3 & 6 & 1630 & 1750 & Compressional \\
\hline
\end{tabular}




\section{Figure Captions}

Fig. 1 (a) The marginal stability boundaries of the antisymmetric ballooning-mirror mode in the equatorial $\left(\beta_{\|}, \mathrm{T}_{\perp} / \mathrm{T}_{\|}\right)$space for $\partial \ln \mathrm{P} / \partial \ln \mathrm{L}=-100,-50,-25,-10,-5,-2.5$, and -1 , respectively, with the fixed parameters: $L=6.6 R_{E}$ and azimuthal mode number $m=50$. The equatorial $\tau=0$ curve is also shown. (b) The same stability boundaries are shown in the equatorial $\left(\alpha_{p}, \tau\right)$ space. Note that for $\tau<0$, the $\alpha_{p}$ threshold is also negative and absolute values of $\alpha_{p}$ for $\tau<0$ are plotted.

Fig. 2 The field-aligned perturbed magnetic field structures of the marginally stable antisymmetric modes along a marginal stability curve in Fig. 8(a) with equatorial values of (a) $T_{\perp} / T_{\|}=1, \beta_{\|}=1.93(\tau=$ 1), (b) $T_{\perp} / T_{\|}=1.3, \beta_{\|}=1.489(\tau=0.419)$, (c) $T_{\perp} / T_{\|}=1.6, \beta_{\|}=0.987(\tau=0.052)$, and (d) $\mathrm{T}_{\perp} / \mathrm{T}_{\|}=2, \beta_{\|}=0.575(\tau=-0.149)$. The fixed parameters are $\mathrm{L}=6.6 \mathrm{R}_{\mathrm{E}}$, azimuthal mode number $\mathrm{m}=5($ ), and $\partial \ln \mathrm{P} / \partial \ln \mathrm{L}=-5$.

Fig. 3 The values of $\tau$ and $\alpha_{p}$ computed from the AMPTE/CCE particle data separately for (a) the transverse wave events and (b) the compressional wave events listed in Table 1. Also plotted are the marginal stability boundary curves of the antisymmetric $\delta B_{\|}$ballooning-mirror modes numerically computed for a local bi-Maxwellian velocity distribution with the pressure gradient in the range of $100>|\partial \ln \mathrm{P} / \partial \ln L|>1$. 

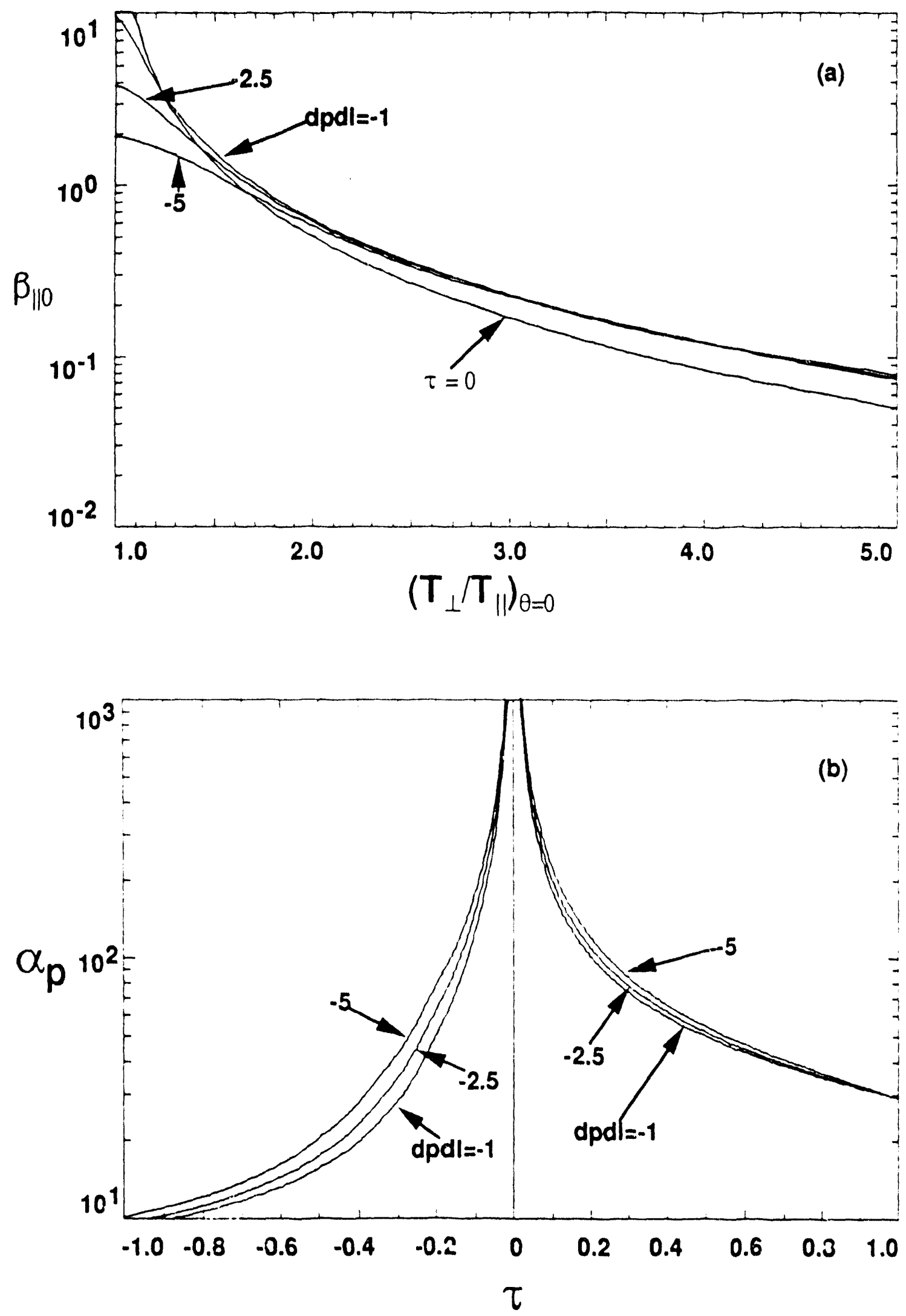

Fig. 1 
$\frac{1}{N} \infty+\infty \quad \infty \quad \infty \vec{N}$

$\frac{\dot{N}}{\infty} \dot{-} 0+\infty \vec{N}$
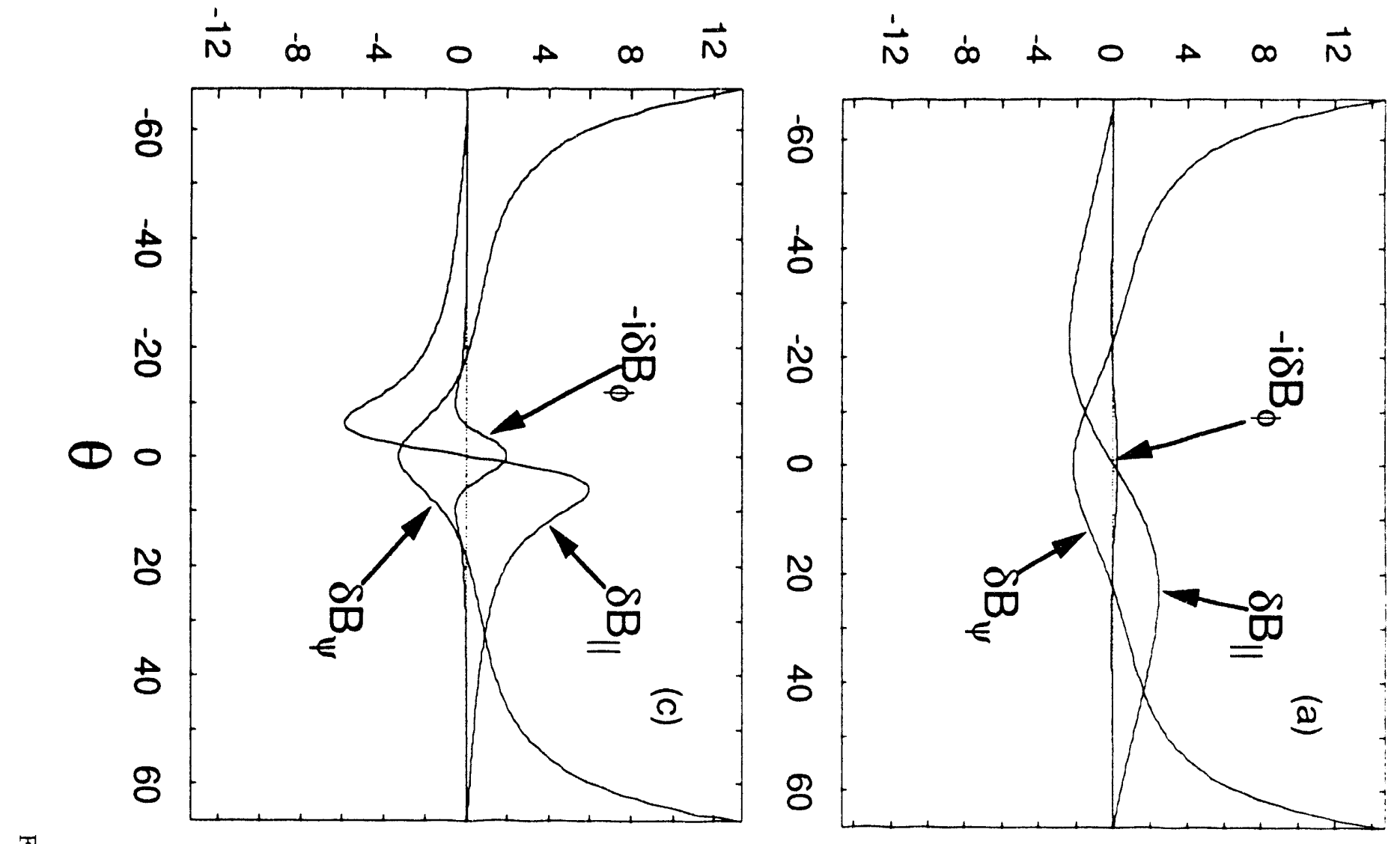

$\underset{\substack{\text { T. } \\ \text { in }}}{\text { ind }}$
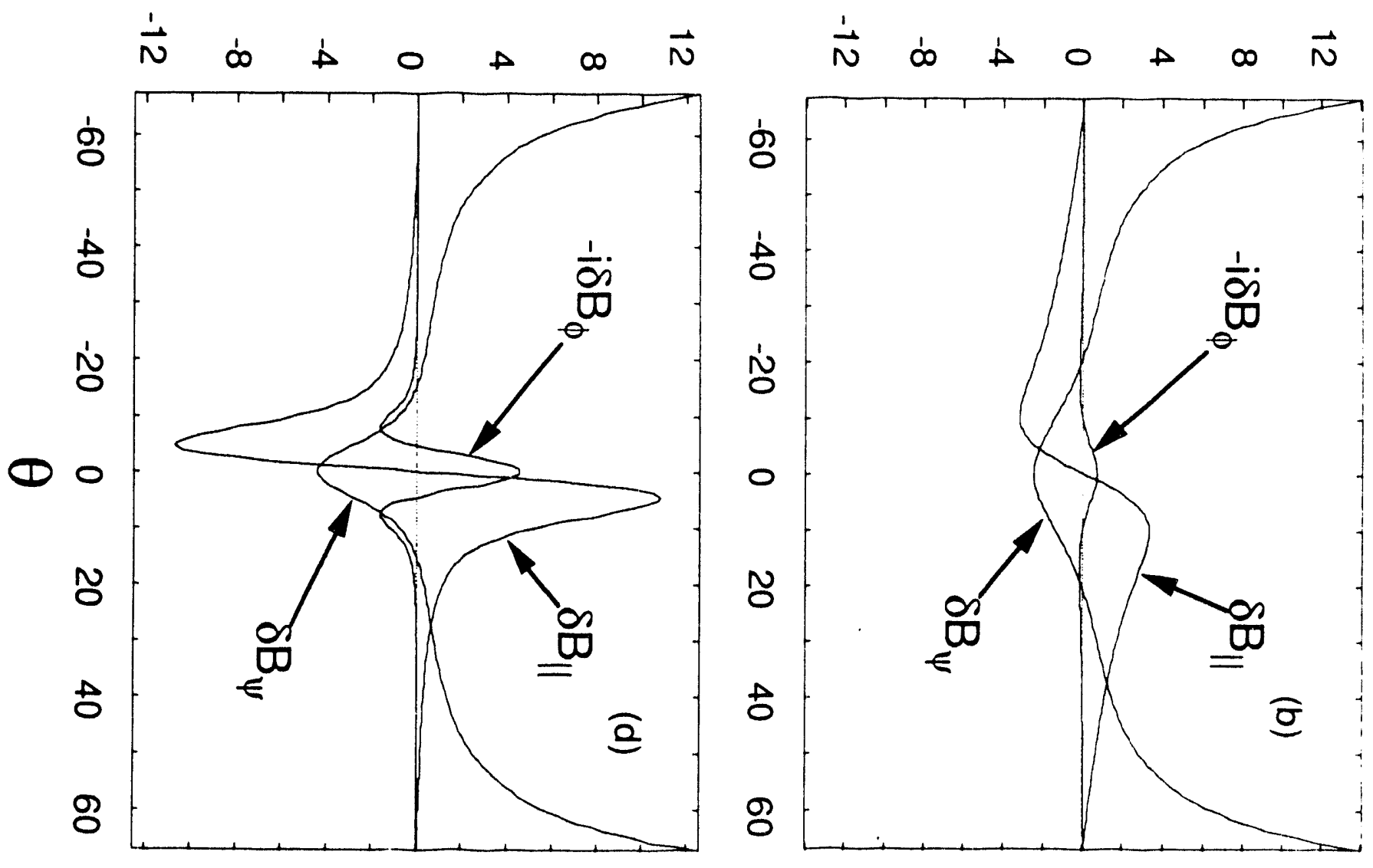


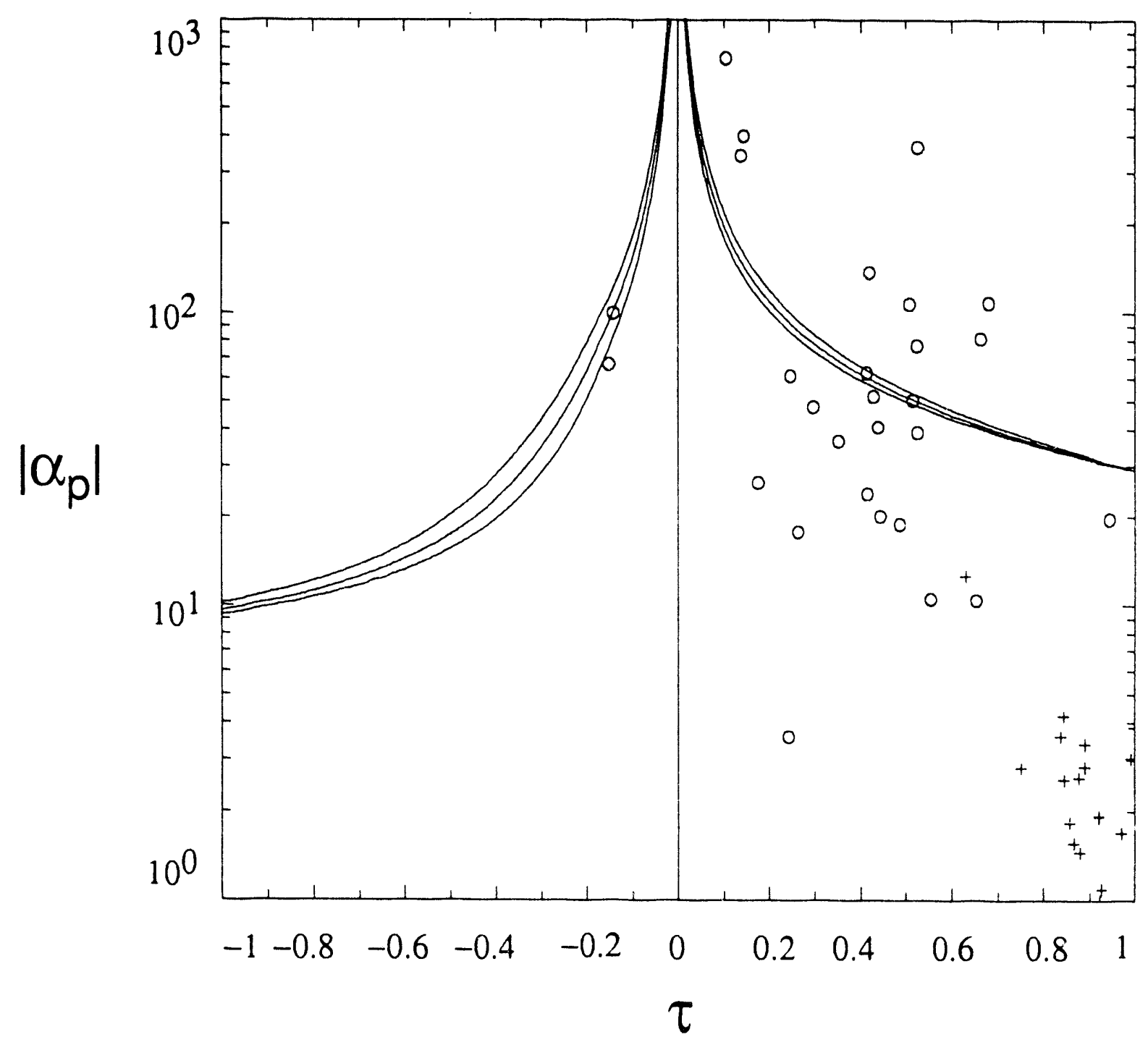

Fig. 3 


\section{EXTERNAL DISTRIBUTION IN ADDITION TO UC-420}

Dr. F. Paoloni, Univ. of Wollongong, AUSTRALIA

Prof. M.H. Brennan, Univ of Sydney, AUSTRALIA

Plasma Research Lab., Australian Nat. Univ., AUSTRALIA

Prof. I.R. Jones, Flinders Univ, AUSTRALIA

Prof. F. Cap, Inst. for Theoretical Physics, AUSTRIA

Prol. M. Heindler, Institut für Theoretische Physik, AUSTRIA

Prof. M. Goossens, Astronomisch Instituut, BELGIUM

Ecole Royale Militaire, Lab. de Phy. Plasmas, BELGIUM

Commission-European, DG. XII-Fusion Prog., BELGIUM

Prof. R. Bouciqué, Rijksuniversiteit Gent, BELGIUM

Dr. P.H. Sakanaka, Instituto Fisica, BRAZIL

Prof. Dr. I.C. Nascimento, Instituto Fisica, Sao Paulo, BRAZIL Instituto Nacional De Pesquisas Espaciais-INPE, BRAZIL

Documents Office, Atomic Energy of Canada Lid., CANADA

Ms. M. Morin, CCFMTokamak de Varennes, CANADA

Dr. M.P. Bachynski, MPB Technologies, Inc., CANADA

Dr. H.M. Skarsgard, Univ. of Saskatchewan, CANADA

Prof. J. Teichmann, Univ, of Montreal, CANADA

Prof. S.R. Sreenivasan, Univ. of Calgary, CANADA

Prof. T.W. Johnston, INRS-Energie, CANADA

Dr. R. Bolton, Centre canadien de fusion magnétique, CANADA

Dr. C.R. James, Univ. of Alberta, CANADA

Dr. P. Lukác, Komenského Universzita, CZECHO-SLOVAKIA

The Librarian, Culham Laboratory, ENGLAND

Library, R61, Rutherford Appleton Laboratory, ENGLAND

Mrs. S.A. Hutchinson, JET Library, ENGLAND

Dr. S.C. Sharma, Univ. of South Pacific, FIJI ISLANDS

P. Mähönen, Univ. of Helsinki, FINLAND

Prof. M.N. Bussac, Ecole Polytechnique, FRANCE

C. Mouttet, Lab. de Physique des Milieux Ionisés, FRANCE

J. Radet, CEN/CADARACHE - Bat 506, FRANCE

Prof. E. Economou, Univ. of Crete, GREECE

Ms. C. Rinni, Univ. of loannina, GREECE

Preprint Library, Hungarian Academy of Sai., HUNGARY

Dr. B. DasGupta, Saha Inst. of Nuclear Physics, INDIA

Dr. P. Kaw, Inst. for Plasma Research, INDIA

Dr. P. Rosenau, Israel Inst. of Technology, ISRAEL

Librarian, International Center for Theo Physics, ITALY

Miss C. De Palo, Associazione EURATOM-ENEA, ITALY

Dr. G. Grosso, Istituto di Fisica del Plasma, ITALY

Prof. G. Rostangni, Istituto Gas lonizzati Del Cnr, ITALY
Dr. H. Yamato, Toshiba Res \& Devel Center, JAPAN

Prof. I. Kawakami, Hiroshima Univ., JAPAN

Prof. K. Nishikawa, Hiroshima Univ., JAPAN

Librarian, Naka Fusion Research Establishment, JAERI, JAPAN

Director, Japan Atomic Energy Research Inst., JAPAN

Prof. S. Itoh, Kyushu Univ., JAPAN

Research Into. Ctr., National Instit. for Fusion Science, JAPAN

Prof. S. Tanaka, Kyoto Univ., JAPAN

Library, Kyoto Univ., JAPAN

Prof. N. Inove, Univ. of Tokyo, JAPAN

Secretary, Plasma Section, Electrotechnical Lab., JAPAN

S. Mori, Technical Advisor, JAERI, JAPAN

Dr. O. Mitarai, Kumamoto Inst. of Technology, JAPAN

Dr. G.S. Lee, Korea Basic Sci. Ctr., KOREA

J. Hyeon-Sook, Korea Alomic Energy Research Inst., KOREA

D.1. Choi, The Korea Adv. Inst. of Sci. \& Tech., KOREA

Prof. B.S. Liley, Univ. of Waikato, NEW ZEALAND

Inst of Physics, Chinese Acad Sci PEOPLE'S REP. OF CHINA

Library, Inst. of Plasma Physics, PEOPLE'S REP. OF CHINA

Tsinghua Univ. Library, PEOPLE'S REPUBLIC OF CHINA

Z. Li, S.W. Inst Physics, PEOPLE'S REPUBLIC OF CHINA

Prof. J A.C. Cabral, Instituto Superior Tecnico, PORTUGAL

Prot. M.A. Hellberg, Univ. of Natal, S. AFRICA

Prot. D.E. Kim, Pohang Inst. of Sci. \& Tech., SO. KOREA

Prof. C.I.E.M.A.T, Fusion Division Library, SPAIN

Dr. L. Stenflo, Univ. of UMEA, SWEDEN

Library, Royal Inst. of Technology, SWEDEN

Prof. H. Wilheimson, Chalmers Univ. of Tech., SWEDEN

Centre Phys. Des Plasmas, Ecole Polytech, SWITZERLAND

Bibliotheek, Inst. Voor Plasma-Fysica, THE NETHERLANDS

Asst. Prof. Dr. S. Cakir, Middle East Tech. Univ., TURKEY

Dr. V.A. Glukhikh,Sci. Res. Inst. Electrophys.I Apparatus, USSR

Dr. D.D. Ryutov, Siberian Branch of Academy of Sci., USSR

Dr. G.A. Eliseev, I.V. Kurchatov Inst., USSR

Librarian, The Ukr.SSR Academy of Sciences, USSR

Dr. L.M. Kovrizhnykh, Inst. of General Physics, USSR

Kernforschungsanlage GmbH, Zentraibibliothek, W. GERMANY Bibliothek, Inst. Für Plasmaforschung, W. GERMANY

Prof. K. Schindler, Ruhr-Universitát Bochum, W. GERMANY Dr. F. Wagner, (ASDEX), Max-Planck-Institut, W. GERMANY Librarian, Max-Planck-Institut, W. GERMANY 

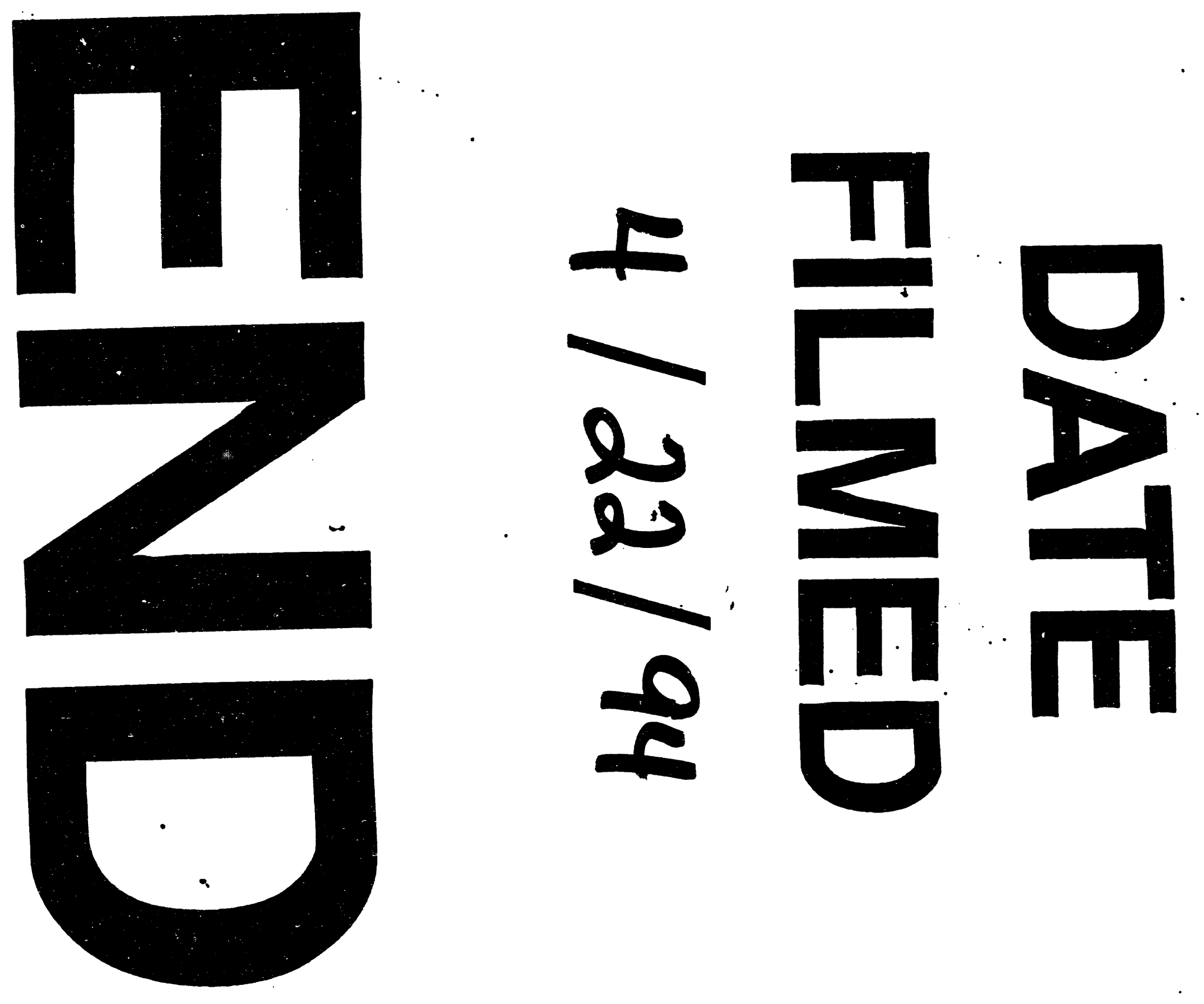


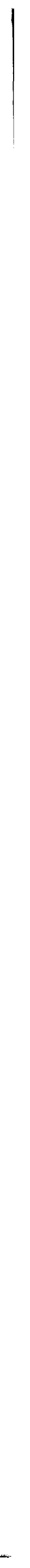

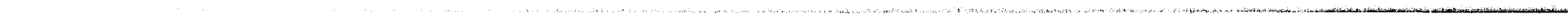

